\title{
Inflammatory biomarkers do not distinguish between patients with sciatica and referred leg pain within a primary care population: results from a nested study within the ATLAS cohort
}

\author{
Samantha L. Hider ${ }^{1,2^{*}}$ D, Kika Konstantinou ${ }^{1,2}$, Elaine M. Hay ${ }^{1}$, John Glossop ${ }^{2}$ and Derek L. Mattey ${ }^{1,2}$
}

\begin{abstract}
Background: There is increasing interest in the role of pro-inflammatory cytokines in the pathogenesis of sciatica and whether these could be potential targets for treatment. We sought to investigate serum biomarker levels in patients with low back-related leg pain, including sciatica.

Methods: Primary care consulters aged $>18$ with low back-related leg pain were recruited to a cohort study (ATLAS). Participants underwent a standardised clinical assessment, lumbar spine MRI and a subsample $(n=119)$ had samples taken for biomarker analysis. Participants were classified having: a) clinically confirmed sciatica or referred leg pain, and then subdivided into those with (or without) MRI confirmed nerve root compression due to disc prolapse. Seventeen key cytokines, chemokines and matrix metalloproteinases (MMPs) implicated in sciatica pathogenesis including TNFa and IL-6, were assayed in duplicate using commercial multiplex detection kits and measured using a Luminex suspension array system. Median biomarker levels were compared between the groups using a Mann Whitney $U$ test. Multivariate logistic regression analysis was used to investigate the association between clinical measures and biomarker levels adjusted for possible confounders such as age, sex, and symptom duration.

Results: No difference was found in the serum level of any of the 17 biomarkers tested in patients with $(n=93)$ or without $(n=26)$ clinically confirmed sciatica, nor between those with $(n=44)$ or without $(n=49)$ sciatica and MRI confirmed nerve root compression.

Conclusion: In this cohort, no significant differences in serum levels of TNFa, IL-6 or any other biomarkers were seen between patients with sciatica and those with back pain with referred leg pain. These results suggest that in patients with low back-related leg pain, serum markers associated with inflammation do not discriminate between patients with or without clinically confirmed sciatica or between those with or without evidence of nerve root compression on MRI.
\end{abstract}

Keywords: Sciatica, Low back pain, Cytokines, TNFa, Radiculopathy

\footnotetext{
* Correspondence: s.hider@keele.ac.uk

${ }^{1}$ Arthritis Research UK Primary Care Centre, Research Institute of Primary Care and Health Sciences, Keele University, Keele, Staffordshire ST5 5BG, UK

${ }^{2}$ Midlands Partnership Foundation Trust, Haywood Hospital, Stoke-on-Trent,

Staffordshire ST6 7AG, UK
}

(c) The Author(s). 2019 Open Access This article is distributed under the terms of the Creative Commons Attribution 4.0 International License (http//creativecommons.org/licenses/by/40/) which permits unrestricted use, distribution, and reproduction in any medium, provided you give appropriate credit to the original author(s) and the source, provide a link to the Creative Commons license, and indicate if changes were made. The Creative Commons Public Domain Dedication waiver (http://creativecommons.org/publicdomain/zero/1.0/) applies to the data made available in this article, unless otherwise stated. 


\section{Background}

Lumbar spinal radicular pain (with or without radiculopathy) or sciatica, represents one distinct presentation of low back-related leg pain, which is often characterised by pain radiating to below the knee and into the foot. Patients with sciatica suffer more severe pain and disability and take longer to recover than those with low back pain (LBP) alone [1]. Although traditionally initially thought to be caused by mechanical nerve root compression, usually by a herniated disc, studies suggest inflammation (mediated by cytokines) also contributes to the pathogenesis of sciatica [2].

Interest on possible inflammatory aetiologies for sciatica initially focused on the cytokine TNF $\alpha$, including randomised trials of anti-TNF inhibitors infliximab [3] and adalumimab [4], showing some improvement in leg pain and reduction in numbers of patients needing spinal surgery [4], with meta-analyses suggesting further trials are warranted [5-7]. These therapies may represent a novel way to treat sciatica but are expensive, and given that sciatica is common, data is required to effectively target these drugs at patients more likely to derive benefit.

Other pro-inflammatory cytokines investigated include IL-6- with studies showing high levels of IL-6 in disc samples from patients undergoing surgery [8]. A study by Pederson et al. [9] showed higher serum levels of IL-6 and IL- 8 in patients with persistent sciatic pain, defined as pain levels on a Visual Analogue Score (VAS) of $>3$, compared to those with pain levels on VAS of $<3$ at 12 months. A second study by Wang et al. [10] also showed higher levels of IL-6, IL-8, TNF $\alpha$ and IL-4 in patients with severe sciatica (defined as pain VAS >3) compared to those with mild sciatica (VAS $<3$ ) or healthy controls. However patients with severe sciatica had a long duration of symptoms (mean (SD) duration 48 (28.96) weeks) and were recruited from a secondary care spinal clinic and thus it is not clear whether these elevated biomarker levels represent patients with chronic or severe symptoms or whether these biomarker levels are elevated in a less selected population. Such an approach would help further elucidate whether these biomarkers are elevated early in the symptom course or could be used in predicting outcome or guide treatment for sciatic pain.

Therefore, the aim of this study was to examine serum levels of key biomarkers in patients presenting in primary care with symptoms of low back-related leg pain including sciatica, and to see whether levels were different in patients with clinically diagnosed sciatica with or without or evidence of nerve root compression on MRI.

\section{Methods}

\section{Participants}

Primary care consulters aged $>18$ with low back-related leg pain were recruited as part of a longitudinal study (the ATLAS study) investigating the overall prognosis of low back-related leg pain and sciatica in primary care. The ATLAS study procedures have been described in detail elsewhere [11], here we give brief information on recruitment and assessment details. Patients consulting their GP (General Practitioner) low back and leg pain, who were potentially eligible for the ATLAS study, were sent a letter with information about the study, an invitation to attend the research clinic, and baseline questionnaires capturing sociodemographic, pain, psychological and health variables. Patients with other inflammatory conditions, such as rheumatoid arthritis were excluded. At the research clinic, patients underwent a standardised clinical assessment by one of seven experienced musculoskeletal physiotherapists, and were diagnosed as having clinically defined sciatica (spinal nerve root involvement) or referred (non-specific) leg pain, based on the examiner's clinical opinion. Providing there were no clinical contraindications to the procedure, patients had a lumbar spine magnetic resonance imaging (MRI) scan within 2 weeks of their baseline assessment. The MRI findings were not part of the clinical diagnosis of sciatica or referred leg pain. All MRIs were scored by a single assessor, a senior consultant musculoskeletal radiologist. The assessor provided a clinical report indicating the presence/absence of definite or possible nerve root compression by lumbar spinal level (3 lower lumbar levels) and side (right/left) and the reason(s) for it if present (e.g. disc prolapse, stenosis) as per normal practice in radiological reporting.

For the purposes of this nested study (which for practical considerations, i.e. availability of phlebotomy service and storage for blood samples, could only be conducted at a single research site), patients consenting to the main study at this site, were also invited to take part in the biomarker sub study. Consenting patients had serum samples taken for biomarker analysis in addition to the standardised clinical assessment.

Participants were classified as having nerve root involvement due to suspected disc prolapse on the basis of the clinical assessment (clinically defined: yes/no).

For the purposes of analysis, we first examined levels of serum biomarkers between patients with clinically diagnosed disc-related sciatica and with referred leg pain, and secondly we examined levels of serum biomarkers between patients with clinically diagnosed disc-related nerve root involvement and evidence of 
concordant MRI findings, and those patients without evidence of concordant MRI findings.

All patients provided written informed consent. The biomarker study was approved by the North West- Greater Manchester South Research Ethics Committee (REC reference number: 12/NW/0173).

\section{Measurement of cytokines, chemokines and MMPs}

Sera were separated from bloods collected in plain BD Vacutainer ${ }^{\circ}$ tubes at study entry. All sera were stored at $-70^{\circ}$ $\mathrm{C}$ until required. Key cytokines, chemokines and matrix metalloproteinases (MMPs) implicated in sciatica pathogenesis including TNF $\alpha$, IL-1, IL-6, MMP1,3,8, aggregan and monocyte chemoattractant protein-1 (MCP-1/CCL-2) were measured in duplicate on a Bio-Plex ${ }^{\mathrm{Tm}} 200$ suspension array system using commercially available multiplex detection kits for cytokines (Milliplex multi-analyte profiling (MAP) kits, Millipore (UK) Ltd., Walford, Hertfordshire, UK), or Fluorokine MAP kits for MMPs (R\&D Systems Europe, Abingdon, Oxfordshire, UK). Further details on the kits used are available in the Appendix. All assays were carried out according to the manufacturers' instructions. High and low control samples were used in each assay.

\section{Statistical analysis}

All data were tested for normality and the appropriate parametric or non-parametric test was selected for analysis. Initial univariable analysis was carried out using the Mann Whitney $U$ test to compare median cytokine levels between groups. A multivariate variable selection procedure using the algorithm of McHenry [12] was used to select biomarker variables which showed the strongest association with either clinically determined sciatica, with/without MRI evidence of nerve root compression. Multivariate logistic regression analysis was used to investigate the association between clinical measures and biomarker levels while adjusting for possible confounders such as age, sex, and symptom duration.

Statistical analyses were carried out using Number Cruncher Statistical Software package for Windows (NCSS 2000, NCSS Statistical Software, Kaysville, Utah, USA). The significance level was set at a $p$ value of 0.05 .

\section{Results}

Data for the main ATLAS cohort are published elsewhere [13]. One hundred nineteen patients were recruited to the biomarker sub study, of these 73 (61.2\%)

Table 1 Comparison of key characteristics for patients clinically diagnosed with or without sciatica. All figures are frequencies (percentages) unless stated otherwise as mean (SD)

\begin{tabular}{|c|c|c|c|}
\hline \multirow[t]{2}{*}{ Characteristics } & \multicolumn{2}{|c|}{$\begin{array}{l}\text { Clinically defined sciatica } \\
n=93\end{array}$} & \multirow[t]{2}{*}{$\begin{array}{l}\text { Referred pain } \\
n=26\end{array}$} \\
\hline & $\begin{array}{l}M R I+v e \\
(n=44)\end{array}$ & $\begin{array}{l}\text { MRI -ve } \\
(n=49)\end{array}$ & \\
\hline \multicolumn{4}{|l|}{ Socio-demographics } \\
\hline Age (years), mean (SD) & $55.2(11.7)$ & $50.3(14.7)$ & $49.5(11.1)$ \\
\hline Female gender, $N(\%)$ & $24(55)$ & $32(65)$ & $17(65)$ \\
\hline BMI, mean (SD) & $30.8(6.3)$ & $29.5(6.2)$ & $32.5(8.2)$ \\
\hline Current smoker, N (\%) & $17(39)$ & $14(28)$ & $9(34)$ \\
\hline \multicolumn{4}{|l|}{ Pain } \\
\hline RMDQ disability score (0-23), mean (SD) & $12.5(5.1)$ & $11.8(6.0)$ & $11.8(6.8)$ \\
\hline Back pain intensity NRS, mean (SD) & $6.6(2.3)$ & $6.7(2.2)$ & $6.9(2.3)$ \\
\hline Leg pain intensity, NRS, mean (SD) & $6.4(2.4)$ & $6.1(2.4)$ & $7.0(2.4)$ \\
\hline Pain below knee, $N(\%)$ & $30(71)$ & $28(61)$ & $21(84)$ \\
\hline Leg pain is worse, $N(\%)$ & $21(49)$ & $22(45)$ & $16(61)$ \\
\hline \multicolumn{4}{|l|}{ Duration of current symptoms ${ }^{a}$} \\
\hline \multicolumn{4}{|l|}{ Back pain } \\
\hline Back pain< 6 weeks, $N(\%)$ & $15(36)$ & $14(29)$ & $7(27)$ \\
\hline Back pain 6-12 weeks, N (\%) & $12(29)$ & $8(17)$ & $7(27)$ \\
\hline Back pain > 3 months, $N(\%)$ & $15(36)$ & $26(54)$ & $12(46)$ \\
\hline \multicolumn{4}{|l|}{ Leg pain } \\
\hline Leg pain< 6 weeks, $N(\%)$ & $17(40)$ & $15(33)$ & $8(32)$ \\
\hline Leg pain 6-12 weeks, $N(\%)$ & $15(36)$ & $9(20)$ & $8(32)$ \\
\hline Leg pain> 3 months, $N(\%)$ & $10(24)$ & $22(48)$ & $9(36)$ \\
\hline
\end{tabular}

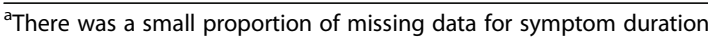


were female. Ninety three $(78 \%)$ were clinically diagnosed with sciatica and of these, 44 (47.3\%) had MRI confirmed nerve root compression due to disc prolapse. Table 1 shows the baseline demographics of the cohort.

None of the biomarkers tested showed any difference in level between patients with or without clinically confirmed sciatica, nor did they show any differences between sciatica patients with MRI findings of nerve root compression $(n=44)$ and those without $(n=49)$. Serum IL-6 levels were low or below the level of detection, and no difference was seen in the levels of IL- 6 or TNF $\alpha$ or any of the other biomarkers between the groups (Table 2). None of the biomarkers selected were significantly associated with either clinically determined sciatica or sciatica with positive MRI findings, in the multivariate variable selection procedure or logistic regression analysis. There was no evidence of significant multicollinearity.

Table 2 Median biomarker levels in those with clinically defined sciatica and in those with and without MRI findings

\begin{tabular}{|c|c|c|c|c|}
\hline \multirow[t]{2}{*}{ Biomarker } & \multicolumn{2}{|c|}{ Clinically defined sciatica } & \multicolumn{2}{|c|}{$\begin{array}{l}\text { Clinically defined sciatica plus } \\
\text { MRI changes }\end{array}$} \\
\hline & No $(n=26)$ & Yes $(n=93)$ & No $(n=49)$ & Yes $(n=44)$ \\
\hline TNFa & $11.9(9.7-13.8)$ & $10.7(1.0-14.8)$ & $12.39(1.0-14.74)$ & $9.86(1.0-14.96)$ \\
\hline $\mathbb{I L}-1 \beta$ & BLD & BLD & BLD & BLD \\
\hline IL-6 & BLD & BLD & $4.96(1.6-7.4)$ & $4.21(0.5-7.7)$ \\
\hline IL-33 & $9.0(7.2-10.9)$ & $8.0(6.9-10.4)$ & $9.1(7.9-10.1)$ & $8.8(7.3-10.1)$ \\
\hline IFNY & BLD & BLD & BLD & BLD \\
\hline \multirow[t]{2}{*}{ TNFRI } & 5117.1 & 5087.4 & 5059.9 & 5190.3 \\
\hline & $(4379.9-5692.6)$ & $(4525.4-6033.3)$ & $(4660.4-5838.9)$ & $(4418.2-6113.9)$ \\
\hline \multirow[t]{2}{*}{$|L-1 R|$} & 1488.8 & 1469.6 & 1490.5 & 1423.9 \\
\hline & $(1311.8-1620.9)$ & $(1273.0-1626.3)$ & $(1346.4-1615.8)$ & $(1143.8-1654.0)$ \\
\hline \multirow[t]{2}{*}{$\|L-1 R\|$} & 6913.9 & 6985.1 & 7347.6 & 6797.3 \\
\hline & $(5541.3-7819.7)$ & (6099.9-8066.3) & $(6513.9-8414.0)$ & $(5818.4-7825.0)$ \\
\hline \multirow[t]{2}{*}{ IL-1Ra } & 866.3 & 998.4 & 974.4 & 1019.7 \\
\hline & $(575.8-1267.8)$ & (735.8-1421.6) & $(693.9-1419.7)$ & $(7417.7-1508.9)$ \\
\hline \multirow[t]{2}{*}{ CXCL8 } & 2.8 & 3.6 & 3.7 & 4.7 \\
\hline & $(0.9-3.5)$ & $(1.5-6.4)$ & $(1.7-6.5)$ & $(2.3-7.1)$ \\
\hline \multirow[t]{2}{*}{ MCP-1 } & 313.7 & 257.9 & 274.3 & 310.0 \\
\hline & $(247.7-313.7)$ & $(207.8-361.8)$ & $(191.1-348.2)$ & $(213.6-416.0)$ \\
\hline FGF $\beta$ & BLD & BLD & BLD & BLD \\
\hline VEGF & $42.9(19.4-62.2)$ & $65.0(22.0-98.5)$ & $66.2(27.6-96.9)$ & $63.5(20.7-123.8)$ \\
\hline \multirow[t]{2}{*}{ HGF } & 265.5 & 245.9 & 244.9 & 245.9 \\
\hline & $(212.5-351.9)$ & $(191.0-321.7)$ & $(182.6-315.1)$ & $(196.9-315.1)$ \\
\hline \multirow[t]{2}{*}{ Ang-2 } & 2164.8 & 2465.0 & 2311.2 & 2870.0 \\
\hline & $(1637.7-3385.0)$ & $(1842.1-3375.4)$ & (1659.9-3070.6) & $(1921.6-3456.1)$ \\
\hline \multirow[t]{2}{*}{ MMP-1 } & 8450.0 & 7857.2 & 7344.9 & 8011.0 \\
\hline & $(4267.3-13,173.5)$ & $(5031.0-15,134.3)$ & $(5113.1-12,124.3)$ & $(4171.7-24,762.6)$ \\
\hline \multirow[t]{2}{*}{ MMP-2 } & $46,778.4$ & $44,214.2$ & $42,714.7$ & $44,418.4$ \\
\hline & $(45,258.0-48,596.9)$ & $(37,585.8-50,496.4)$ & $(36,594.9-50,419.9)$ & $(38,275.4-50,668.8)$ \\
\hline \multirow[t]{2}{*}{ MMP-3 } & $13,741.5$ & $12,111.6$ & $12,852.8$ & $11,882.8$ \\
\hline & $(8626.9-22,480.1)$ & $(8403.3-18,651.9)$ & $(7733.5-18,337.4)$ & (8863.1-18,789.5) \\
\hline \multirow[t]{2}{*}{ MMP-8 } & 8942.1 & 7409.5 & 7842.8 & 6091.0 \\
\hline & $(4098.4-11,552.5)$ & $(4509.8-12,196.8)$ & $(4518.1-10,694.5)$ & $(4181.4-13,098.2)$ \\
\hline \multirow[t]{2}{*}{ Aggrecan } & 943.0 & 922.9 & 911.2 & 961.4 \\
\hline & $(747.4-1302.5)$ & $(618.8-1253.4)$ & $(624.7-1272.3)$ & (589.5-1243.3) \\
\hline
\end{tabular}




\section{Discussion}

LBP and sciatica are common health problems with significant impact at both the individual level (in terms of pain and disability) and the health and societal level (in terms of healthcare costs and work loss). Studies have suggested a possible inflammatory aetiology in the development or persistence of sciatic pain, and given the availability of cytokine inhibitors for treatment of inflammatory arthritis, this has prompted research to determine whether these agents could be effective for sciatica. Previous studies have confirmed the presence of key cytokines such as TNF $\alpha$ and IL- 6 in surgical disc samples, and recent data have suggested that serum levels of these cytokines may be elevated in patients with sciatica symptoms [8-10], although other studies have shown no association [14].

In this study of patients with low back-related leg pain seeking primary healthcare, no difference was found in the levels of TNF $\alpha$ or IL- 6 between those with or without either clinically defined sciatica or clinically defined sciatica with concordant MRI findings. The results of this study are in contrast to those of Wang [10] who found elevated levels of TNF $\alpha$ and IL-6 in patients with severe sciatica (defined as pain level of $>3$ on VAS). However, in part this difference may be explained by the characteristics of the patients recruited in the study by Wang et al. [10], as patients were recruited from a spinal clinic and had a mean symptom duration of 48 weeks. By contrast, our cohort was recruited from primary care attenders who had a shorter duration of symptoms, with over one third of patients having leg pain duration of less than 6 weeks.

There are a number of strengths and weaknesses that need to be considered when interpreting the results of this study. A key strength is that the cohort represents an unselected population of primary care consulters with low back-related leg pain and sciatica rather than being recruited from spinal surgical clinics and as such the results are likely to be highly generalizable. Furthermore, patients underwent a standardised clinical assessment and MRI scan [11], providing confidence in the likely diagnosis of radicular pain. As all participants in the biomarker sub-study were recruited from a single research clinic, all samples were taken at a similar time of day thus reducing biological variability.

One of the weaknesses is that biomarker measurements were made at a single time point only, so we are not able to determine whether there were fluctuations in the levels of biomarkers over time (associated with the level of pain for example. Other hypotheses that may explain our negative results are that firstly we did not have a group of pain free controls for comparison (albeit that this was not the original aim of the study) or that the groups were not sufficiently different to detect differences. Since there are currently no internationally agreed standard benchmark serum levels for any of these biomarkers, comparisons across studies is not possible [15]. Secondly, given that some of the subgroups were small the study was underpowered to be able to detect differences in biomarker levels between the subgroups. Nevertheless, the unselected primary care population, who underwent standardised assessments, ensures that the cohort is generalizable. Further longitudinal studies are required to determine whether cytokine levels become elevated in those with persistent symptoms rather than those with a short symptom duration.

\section{Conclusion}

In summary, in our primary care cohort of patients with low back-related leg pain including sciatica, serum markers associated with inflammation, angiogenesis or extracellular matrix remodelling do not discriminate between patients with and without sciatica, or between those with sciatica with and without obvious evidence of nerve root compression on MRI. Larger longitudinal studies are required to determine whether any serum biomarker levels are associated with persistence of sciatica symptoms or whether other biomarkers could be important in the pathogenesis and persistence of sciatica.

\section{Appendix}

The Milliplex-MAP cytokine assay from Millipore UK was a custom designed assay based on product HCYTOMAG$60 \mathrm{~K}$ which allows the choice of up to 60 different cytokines. The MMP assay from R\&D Systems was also custom designed using a Fluorokine MAP Base kit for MMPs (cat no LMP000) and individual MMP assays (MMP-1 cat no LMP091, MMP-2 cat no LMP902, MMP-3 cat no LMP513, MMP-8 cat no LMP908).

\section{Abbreviations \\ GP: General Practitioner; IFN: Interleukin; IL: Interleukin; LBP: Low back pain; MCP: Monocyte chemoattractant protein; MMP: Matrix Metalloproteinase; MRI: Magnetic resonance imaging; REC: Research Ethics Committee; \\ SD: Standard deviation; TNF: Tumour necrosis factor; VAS: Visual Analogue Score}

\footnotetext{
Acknowledgements

The authors would like to thank the members of the ATLAS study team, and all participating patients.

Funding

The study was funded by an NIHR/CNO Clinical Lectureship awarded to K. Konstantinou. K. Konstantinou was supported by a HEFCE Senior Clinical Lecturer award. EMH is a NIHR Senior NIHR investigator. This paper presents independent research funded by the National Institute for Health Research (NIHR), under its Programme Grants for Applied Research funding scheme: "Optimal management of spinal pain and sciatica in primary care" (NIHR-RPPG-0707-10131). The views expressed are those of the author(s) and not necessarily those of the NHS, the NIHR or the Department of Health and Social Care. The study funders had no role in the study design; data collection, analysis, or interpretation; in the writing of the paper; or in the decision to submit the paper for publication.
} 


\section{Availability of data and materials}

All data generated or analysed during this study are included in this published article. The data used in this analysis are owned by The Arthritis Research UK Primary Care Centre, to which further queries related to data access may be submitted. The Arthritis Research UK Primary Care Centre has established data sharing arrangements to support joint publications and other research collaborations. Applications for access to anonymised data from our research databases are reviewed by the Centre's Data Custodian and Academic Proposal (DCAP) Committee, and a decision regarding access to the data is made subject to the National Research Ethics Service ethical approval first provided for the study and to new analysis being proposed. Consideration to release data will be undertaken in conjunction with journal publication and/ or funder restrictions that may apply. Further information on our data sharing procedures can be found on the Centre's website (www.keele.ac.uk/pchs/datasharing) or by emailing the Centre's data manager (primarycare.datasharing@keele.ac.uk).

\section{Authors' contributions}

$\mathrm{SH}, \mathrm{KK}, \mathrm{EMH}$ and DLM made substantial contributions to conception and design, KK, JG and DLM made substantial contributions to acquisition of data. DLM was responsible for analysis and interpretation of data. All authors been involved in drafting the manuscript or revising it critically for important intellectual content and have given final approval of the version to be published.

\section{Ethics approval and consent to participate}

The study was approved by the North West- Greater Manchester South Research Ethics Committee (REC reference number: 12/NW/0173) and all patients provided written informed consent.

\section{Consent for publication}

Not applicable.

\section{Competing interests}

The authors declare that they have no competing interests.

\section{Publisher's Note}

Springer Nature remains neutral with regard to jurisdictional claims in published maps and institutional affiliations.

Received: 6 June 2018 Accepted: 30 April 2019

Published online: 10 May 2019

\section{References}

1. Konstantinou K, Hider SL, Jordan JL, Lewis M, Dunn KM, Hay EM. The impact of low back-related leg pain on outcomes as compared with low back pain alone: a systematic review of the literature. Clin J Pain. 2013 Jul:29(7):644-54.

2. Valat JP, Genevay S, Marty M, Rozenberg S, Koes B. Sciatica. Best Pract Res Clin Rheumatol. 2010 Apr;24(2):241-52.

3. Korhonen T, Karppinen J, Paimela L, Malmivaara A, Lindgren KA, Bowman C, Hammond A, et al. The treatment of disc-herniation-induced sciatica with infliximab: one-year follow-up results of FIRST II, a randomized controlled trial. Spine (Phila Pa 1976). 2006;31(24):2759-66.

4. Genevay S, Finckh A, Zufferey P, Viatte S, Balagué F, Gabay C. Adalimumab in acute sciatica reduces the long-term need for surgery: a 3-year follow-up of a randomised double-blind placebo-controlled trial. Ann Rheum Dis. 2012;71(4):560-2.

5. Wang YF, Chen PY, Chang W, Zhu FQ, Xu LL, Wang SL, Chang LY, Luo J, Liu GJ. Clinical significance of tumor necrosis factor-a inhibitors in the treatment of sciatica: a systematic review and meta-analysis. PLoS One. 2014;9(7):e103147

6. Williams NH, Lewis R, Din NU, Matar HE, Fitzsimmons D, Phillips CJ, Sutton A, Burton K, Hendry M, Nafees S, Wilkinson C. A systematic review and meta-analysis of biological treatments targeting tumour necrosis factor a for sciatica. Eur Spine J. 2013;22(9):1921-35.

7. Jing S, Yang C, Zhang X, Wen S, Li Y. Efficacy and safety of etanercept in the treatment of sciatica: a systematic review and meta-analysis. J Clin Neurosci. 2017:44:69-74.

8. Andrade P, Hoogland G, Garcia MA, Steinbusch HW, Daemen MA, VisserVandewalle V. Elevated IL-1 $\beta$ and IL-6 levels in lumbar herniated discs in patients with sciatic pain. Eur Spine J. 2013;22(4):714-20. https://doi.org/10. 1007/s00586-012-2502-x Epub 2012 Sep 27.

9. Pedersen LM, Schistad E, Jacobsen LM, Røe C, Gjerstad J. Serum levels of the pro-inflammatory interleukins $6(\mathrm{IL}-6)$ and $-8(\mathrm{IL}-8)$ in patients with lumbar radicular pain due to disc herniation: a 12-month prospective study. Brain Behav Immun. 2015;46:132-6.

10. Wang K, Bao JP, Yang S, Hong X, Liu L, Xie XH, Wu XT. A cohort study comparing the serum levels of pro- or anti-inflammatory cytokines in patients with lumbar radicular pain and healthy subjects. Eur Spine J. 2016; 25(5):1428-34.

11. Konstantinou K, et al. Clinical course, characteristics and prognostic indicators in patients presenting with back and leg pain in primary care. The ATLAS study protocol. BMC Musculoskelet Disord. 2012;13:4.

12. McHenry CE. Computation of a best subset in multivariate analysis. Appl Stat. 1978:27:291-6.

13. Konstantinou K, Dunn KM, Ogollah R, Lewis M, van der Windt D, Hay EM. ATLAS Study Team. Prognosis of sciatica and back-related leg pain in primary care: the ATLAS cohort. Spine J. 2018;18(6):1030-40.

14. Brisby H, Olmarker K, Larsson K, Nutu M, Rydevik B. Proinflammatory cytokines in cerebrospinal fluid and serum in patients with disc herniation and sciatica. Eur Spine J. 2002 Feb;11(1):62-6.

15. SS, Smith MS, Reda D, Suffredini AF, McCoy JP Jr. Multiplex bead array assays for detection of soluble cytokines: comparisons of sensitivity and quantitative values among kits from multiple manufacturers. Cytometry B Clin Cytom. 2004;61(1):35-9.

\section{Ready to submit your research? Choose BMC and benefit from:}

- fast, convenient online submission

- thorough peer review by experienced researchers in your field

- rapid publication on acceptance

- support for research data, including large and complex data types

- gold Open Access which fosters wider collaboration and increased citations

- maximum visibility for your research: over $100 \mathrm{M}$ website views per year

At BMC, research is always in progress.

Learn more biomedcentral.com/submissions 